\title{
Évolution des images du FLE à l'université : une étude de cas au Portugal
}

\section{Sílvia Melo-Pfeifer et Susana Pinto}

\section{OpenEdition}

Journals

Édition électronique

URL : http://journals.openedition.org/rdlc/2112

DOI : $10.4000 /$ rdlc. 2112

ISSN : 1958-5772

Éditeur

ACEDLE

Édition imprimée

Pagination : 153-171

\section{Référence électronique}

Sílvia Melo-Pfeifer et Susana Pinto, «Évolution des images du FLE à l'université : une étude de cas au Portugal », Recherches en didactique des langues et des cultures [En ligne], 6-1 | 2009, mis en ligne le 01 avril 2009, consulté le 24 avril 2019. URL : http://journals.openedition.org/rdlc/2112 ; DOI : 10.4000/ rdlc. 2112

Ce document a été généré automatiquement le 24 avril 2019

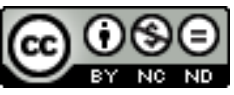

Recherches en didactique des langues et des cultures is licensed under a Creative Commons AttributionNonCommercial-NoDerivatives 4.0 International License 


\title{
Évolution des images du FLE à l'université : une étude de cas au Portugal
}

\author{
Sílvia Melo-Pfeifer et Susana Pinto
}

\section{NOTE DE L'AUTEUR}

Étude soutenue par la Fondation pour la Science et la Technologie, dans le cadre du projet de doctorat de Sílvia Melo et du mémoire de master de Susana Pinto, tous deux en DL et sous la direction scientifique de Maria Helena de Araújo e Sá. Nous remercions Cláudia Ferreira, du Département de Langues-Cultures de l'Université d'Aveiro, pour toutes les corrections et commentaires qu'elle a apportés à cet article.

\section{Introduction}

1 Les images des langues et des cultures qui circulent et se construisent dans des contextes éducatifs sont actuellement au cœur de la recherche en Didactique des Langues (DL) (Androulakis et al., 2007 ; Castellotti \& Moore, 2002 ; Zarate, 1997). Leur étude mobilise des concepts provenant d'autres domaines disciplinaires (en particulier la psychologie sociale et la sociolinguistique) et essaie de décrire le rapport des sujets aux languescultures, ainsi que l'influence de ce rapport dans la communication interculturelle (Yanaprasart, 2002) et dans les processus d'enseignement-apprentissage des langues (Candelier \& Hermann-Brennecke, 1993Küster, 2007).

2 Les images des langues, ainsi que les attitudes et motivations des apprenants, font partie de la dimension socio-affective de l'enseignement-apprentissage des Langues Étrangères (LE). Ces images, attitudes et stéréotypes se développent à travers des interactions sociales (Coste, 1997), notamment à l'école et dans la salle de classe (De Pietro \& Müller, 1997), et jouent un rôle important dans l'acquisition des savoirs et dans la construction et le maintien 
de rapports avec l'Autre (Yanaprasart, 2002). De ce point de vue, il est nécessaire de les faire émerger et de les étudier, afin de mieux comprendre les facteurs qui sont à leur origine et président à leur changement, notamment en contexte universitaire, lieu de contacts plurilingues et interculturels (en particulier à travers la mobilité des étudiants).

Ceci dit, nous considérons, comme Tschoumy (1997: 11), que chacun d'entre nous se construit des images des langues et que "cette représentation entraînera plus ou moins de faveur de tolérance, ou d'élan pour une langue" et pour son apprentissage. Ainsi, cette étude revêt un intérêt particulier dans un moment où l'enseignement-apprentissage du FLE est en régression au Portugal $^{2}$.

4 Ainsi, après une approche du concept central qui soutient ce texte - celui d'image (pris en tant que synonyme de représentation) - ainsi que de sa rentabilité en DL, nous chercherons à expliciter le contenu sémantique des représentations d'étudiants portugais, recueillies à travers des enquêtes, par rapport au Français Langue Étrangère, à deux moments de leur parcours académique: la première (année 2001/2002) et la troisième année (année 2003-2004) curriculaires. Nos conclusions, renvoyant à des images nombreuses mais fortement scolarisées et homogènes de cette langue, nous permettent d'esquisser des considérations sur le poids du cursus universitaire dans la construction et l'évolution de ces images et sur le besoin d'ouverture au plurilinguisme du discours de l'université.

\section{Place des images dans la didactique des langues- cultures}

5 Le concept d'image, souvent pris comme synonyme de représentation, s'assume en tant qu'objet d'étude des chercheurs qui se penchent sur les images par rapport aux langues, leur apprentissage et leurs locuteurs, s'intéressant soit aux apprenants, soit encore aux professeurs/formateurs et aux chercheurs.

6 Dans ces études, le concept est interrogé et (re)défini (Castellotti \& Moore, 2002 ; Mariko, 2005), surtout à cause de sa plasticité et de l'apparence caméléonesque qu'il revêt dans les discours qui le prennent en tant qu'objet :

Le concept de représentation est sans doute un des plus "nomade", passant depuis plusieurs siècles de discipline en discipline avec à chaque fois de nouvelles définitions ou des étiquettes variées comme celles de "conception", "idée", "concept ", "image", "perception", "image", "attitude", "jugement". Or plus un concept est employé, plus il est élastique (Billiez, 1994).

7 Malgré cette indéfinition ou profusion de concepts analogues, le potentiel des images dans l'enseignement-apprentissage des langues n'est pas mis en cause (Dabène, 1997 ; Deprez, 1997 ; Vasseur \& Grandcolas, 1997 ; Matthey, 1997 ; Moore, 2001 ; Paganini, 1994), soit en tant que moteur soit en tant que frein ou obstacle pédagogique :

Les représentations doivent donc être élucidées dès le départ car elles constituent des filtres qui peuvent limiter voire entraver les perceptions, les motivations et les comportements. Elles peuvent diminuer les rendements didactiques, il est donc nécessaire de les faire émerger pour que les sujets améliorent la mobilisation de l'ensemble de leurs savoirs acquis (...) (Billiez, 1994).

Zarate définit le concept d'image en tant que "produit d'un travail social collectif, à travers lequel les agents sociaux construisent leurs modes de connaissance de la réalité" (1993: 29). De leur côté, Castellotti, Coste \& Moore (2001) mettent en évidence la construction sociale des images, les définissant en tant que procès dynamiques, évolutifs, créés par les 
individus et par les groupes à travers des expériences de vie, notamment les contacts interculturels et les expériences d'enseignement-apprentissage.

Se co-construisant dans l'interaction, les images des langues sont des outils sémiotiques et cognitifs et, de ce fait, elles peuvent se transformer et être transmises au cours des dynamiques interactionnelles. Dans ce sens, les images sont influencées par la configuration situationnelle et par les ingrédients du contexte (locuteurs, langues en présence...), ainsi que par un assez grand nombre d'autres facteurs sociolinguistiques (rapport entre les langues en présence, par exemple), culturels, scolaires, économiques et politiques (Araújo e Sá \& Pinto, 2006).

10 Ces définitions et caractéristiques nous laissent entrevoir que les images des langues se situent parmi les conditions (voire ingrédients) qui encadrent l'enseignement-apprentissage, les procès d'appropriation et de formation linguistique. Ainsi, dans le cas des professeurs, les images influencent leurs pratiques professionnelles, le choix des matériaux en salle de classe et la gestion de l'interaction (Cazals-Quenouille, 1995 ; Müller \& De Pietro, 2001). Pour ce qui est des apprenants, les images influencent le choix des langues à apprendre, les stratégies d'apprentissage déployées et la motivation pour s'engager dans les activités de salle de classe ou dans des interactions où les langues s'assument en tant que lieu de construction de rapports inter-individuels (Billiez, 1996; Dabène, 1994; Melo, 2006 ; Monnanteuil, 1994).

11 D'un autre côté, les images sont fortement déterminées par les pratiques de formation et par les pratiques scolaires. À titre d'illustration, nous pouvons signaler que les pratiques d'évaluation peuvent conditionner l'image des compétences développées; les pratiques interactionnelles peuvent influencer la perception de ce qui est valorisé dans une langue ; la survalorisation de l'une des composantes d'une langue peut se décliner dans la perception de la langue dans sa totalité (par exemple, le poids excessif de la grammaire allemande dans la salle de classe au Portugal fait que la langue est perçue comme difficile, Schmidt \& Araújo e Sá, 2005).

12 De ce point de vue, le rôle des images dans l'enseignement-apprentissage des LE met en évidence le besoin de les prendre en compte dans la salle de classe et justifie ainsi leur prise en compte par la recherche en DL. En outre, la nature évolutive et dynamique de ces objets sémiotiques et cognitifs justifie et rend pertinente leur étude diachronique, encore assez rare dans la littérature en DL.

\section{3. Évolution des images de la langue française à l'université : une étude de cas au Portugal}

D'autres études se sont penchées sur l'image du FLE (par exemple, Candelier \& HermannBrennecke, 1993 ; Graham, 2003 ; Verdelhan-Bourgade, 1999; Williams, Burden \& Kanvers, 2002), notamment en contexte allemand (Küster, 2007 ; Androulakis et al., 2007). Dans ces études, le français est perçu surtout en tant qu'objet curriculaire et/ou affectif, auprès des différents publics concernés. À ce propos, Küster, se rapportant aux motivations des lycéens allemands pour apprendre le FLE, observe :

114 Jugendliche nannten den Pflichtaspekt. Sie hoben hervor, dass an ihrer Schule keine wirklichen Alternativen für sie bestanden hätten; 19-mail spielte dis ästhetische Attraktivität der französischen Sprache eine Rolle, und in 7 Fällen hatten die Befragten französische Familienangehörige. 6 Mal wurde die 
Entscheidung für Französisch als günstig für die gymnasiale Laufbahn angesehen (...). Jeweils nur einmal kam die Antwort, Französisch sei leichter als Latein oder habe Ähnlichkeit mit dem Arabischen (sic) $(2007: 213)$.

14 Notre but, dans cette section, est de confronter ces résultats avec ceux que nous avons obtenus auprès d'un public universitaire portugais, rendant encore compte de l'évolution de ces images le long du parcours académique.

\subsection{Public, configuration méthodologique de l'étude et outils de recueil de données}

15 Notre étude s'inscrit dans un projet plus élargi ("Images des langues dans la communication interculturelle" ${ }^{3 "}$, coordonné par Maria Helena de Araújo e Sá de l'Université d'Aveiro Portugal), dont le but était de décrire le contenu sémantique et la circulation d'images des langues et des cultures dans des contextes scolaires et formatifs, en milieux associatifs et dans la presse portugaise (Andrade, Araújo e Sá \& Moreira, 2007). Ce travail se penche, comme annoncé, sur les images du FLE d'étudiants universitaires lusophones en licence "Langues et Relations d'Entreprises" de l'Université d'Aveiro.

16 Cette licence avait une durée totale de 8 semestres et nous avons recueilli les données ici présentées à deux moments de la formation : les années 2001-2002 (première année de la licence) et 2003-2004 (troisième année). Pour ce qui est du curriculum linguistique de cette licence, il faut préciser qu'il intégrait les matières d'Anglais V/VI - Techniques d'expression, Langue B (allemand ou français) et Langue C V/VI (chinois) - Langue et culture. À partir de l'année 2004-2005, ce curriculum proposait, outre l'allemand et le français, l'espagnol en tant que Langue $\mathrm{B}$, et le chinois et l'arabe comme troisième langue.

Le but de cette étude n'est pas seulement de décrire les images que les sujets possèdent du FLE, mais aussi d'essayer de comprendre la façon dont elles évoluent, ainsi que de dégager les agents de cette évolution, dans une licence où les LE et la Gestion sont les composantes principales. Comme nous l'avions affirmé à une autre occasion:

As they are studying four foreign languages, they are a particularly interesting public in terms of trying to understand the relationship between learning environment, languages of study and personal linguistic and communicative profiles of these students (Araújo e Sá, Melo \& Pinto, 2004).

Dans la première année de l'enquête (2001-2002), nous avons demandé aux 38 étudiants de remplir un questionnaire organisé en quatre parties (Araújo e Sá \& Melo, 2005) :

- Moi et les langues étrangères, par laquelle on voulait cerner la biographie et les projets linguistiques des sujets ainsi que le degré de connaissance qu'ils croyaient posséder dans les différentes langues ;

- Moi et les langues romanes, destinée à vérifier leurs connaissances, représentations, attitudes et stéréotypes à l'égard de cette famille linguistique. Les apprenants pouvaient classer les Langues Romanes (LR) au niveau de leur proximité par rapport au portugais et de leur degré de facilité en ce qui concerne la compréhension orale et écrite ainsi que l'interaction verbale. En plus, ils pouvaient aussi caractériser, soit les LR, soit leurs locuteurs, et émettre des opinions sur les avantages/inconvénients de l'apprentissage d'une langue voisine ;

- Moi et l'apprentissage des langues, où l'on cherchait à cerner les profils des apprenants, en particulier pour ce qui était des méthodes d'apprentissage et de travail avec les langues ;

- À la découverte des langues romanes, où les apprenants devaient identifier les LR, ayant comme base des textes écrits et oraux. 
Dans la deuxième année (2003-2004), nous avons demandé aux 53 étudiants de remplir un autre questionnaire, organisé en deux parties :

- L'apprentissage de langues étrangères, qui comprenait des questions sur les langues étudiées au long du parcours scolaire, notamment sur des raisons qui avaient amené à la sélection des langues à étudier et sur les situations de contact avec ces langues ;

- Les langues étrangères étudiées, qui ne prenait en compte que les langues étrangères en étude l'allemand, le chinois, le français et l'anglais (situations de contact avec ces langues, pays étrangers visités, importance donnée à chaque langue, facilité/difficulté d'apprentissage et rapport subjectif à ces langues).

20 En plus, dans cette année, nous avons encore demandé aux étudiants de répondre à la question: "Imaginez que vous devriez décrire la langue $\mathrm{X}$ à quelqu'un n'ayant eu aucun contact préalable avec elle. Qu'est-ce que vous lui diriez et pourquoi ?" (voir Pinto, 2005).

21 Nous présenterons, après explicitation des catégories d'analyse (section 3.2.), les résultats concernant les deux phases de cette étude, partant d'un travail exploratoire précédent (voir Araújo e Sá, Melo \& Pinto, 2004), mais en nous rapportant, de façon approfondie, aux résultats concernant le FLE (section 3.3.).

\subsection{Les catégories d'analyse}

Pour ce qui est de l'analyse et du traitement des données, nous avons utilisé les catégories et les sous-catégories présentées dans le tableau 1, conçues dans le cadre du travail de Pinto $(2005)^{4}$ portant sur les images curriculaires des langues étrangères à l'université. Il faut prendre encore en considération que, s'agissant d'une étude sur un phénomène social et cognitif assez complexe, certaines sous-catégories ont des rapports étroits, ne pouvant être comprises qu'à travers une vision holistique et intégrative.

Tableau 1 - Explicitation des catégories d'analyse.

\begin{tabular}{|c|c|c|}
\hline Catégories $^{5}$ & Sous-catégories & Descripteurs associés \\
\hline \multirow[t]{2}{*}{$\begin{array}{l}\text { FLE comme objet } \\
\text { d'appropriation }\end{array}$} & $\begin{array}{l}\text { Facilité/difficulté } \\
\text { d'apprentissage et d'usage }\end{array}$ & $\begin{array}{l}\text { Difficultés par rapport à la compréhension et à } \\
\text { l'expression, orales et écrites ; exigences cognitives par } \\
\text { rapport à la morphologie, à la phonétique, à la syntaxe, } \\
\text { à la sémantique, à la pragmatique et à l'orthographe. }\end{array}$ \\
\hline & $\begin{array}{l}\text { Éloignement/proximité } \\
\text { par rapport à la LM ou à } \\
\text { d'autres LE étudiées }\end{array}$ & $\begin{array}{l}\text { Rapport entre la LM et le FLE, par rapport aux } \\
\text { similitudes et différences objectives (caractéristiques } \\
\text { typologiques) et subjectives (celles ressenties par les } \\
\text { apprenants). }\end{array}$ \\
\hline \multirow[t]{2}{*}{$\begin{array}{l}\text { FLE comme objet } \\
\text { affectif }\end{array}$} & $\begin{array}{l}\text { Rapport affectif } \\
\text { apprenant }- \text { langue - } \\
\text { culture }\end{array}$ & $\begin{array}{l}\text { Plaisir/dégoût provoqué par le contact avec le FLE et } \\
\text { ses locuteurs ; élan et curiosité vis-à-vis des langues- } \\
\text { cultures francophones. }\end{array}$ \\
\hline & Paysage sonore du FLE & $\begin{array}{l}\text { Impressions phonétiques, rythme, intonation, volume, } \\
\text { prononciation (une langue belle ou laide). }\end{array}$ \\
\hline
\end{tabular}




\begin{tabular}{|l|l|l|}
\hline $\begin{array}{l}\text { FLE comme objet } \\
\text { de pouvoir }\end{array}$ & Pouvoir social et culturel & $\begin{array}{l}\text { Utilité du FLE dans l'ascension sociale et culturelle et } \\
\text { dans l'auto promotion personnelle. }\end{array}$ \\
\hline & $\begin{array}{l}\text { Pouvoir économique et } \\
\text { professionnel }\end{array}$ & $\begin{array}{l}\text { Valeur/utilité internationale du FLE dans les échanges } \\
\text { économiques et sur le marché du travail; FLE comme } \\
\text { investissement économique et, donc, comme outil } \\
\text { permettant l'ascension économique et professionnelle. }\end{array}$ \\
\hline
\end{tabular}

\subsection{Analyse et discussion des données : I'influence du parcours scolaire dans la (re)construction des images}

23 Avant de présenter les données concernant les images du FLE, nous présentons un bref profil sociolinguistique et communicatif de ces étudiants, car nous considérons que les images des langues ne peuvent pas être élucidées et comprises en dehors du cadre général auquel elles appartiennent et, plus particulièrement, en dehors des histoires linguistiques des sujets qui les énoncent.

24 À travers le premier questionnaire, nous avons constaté que ces étudiants, avant leur accès à l'université, possédaient des répertoires linguistiques et communicatifs assez réduits et ancrés dans le parcours scolaire, dans la mesure où les LE étudiées se limitaient à l'anglais, au français et à l'allemand (les LE du cursus portugais à cette époque ${ }^{6}$ ). Les contacts informels avec les LE sont aussi réduits et presque limités à la télévision ${ }^{7}$ et à Internet. Par rapport à l'image de leurs compétences en LE, les étudiants ne révèlent pas de conscience en ce qui concerne les possibilités d'intercompréhension entre les langues, notamment de la même famille. En plus, l'image de compétence est fortement ancrée dans les apprentissages et le succès scolaires : être compétent dans une langue (même dans la LM) signifie que la langue a été apprise à l'école et que l'évaluation a été positive à la fin de l'année.

Par rapport à leur culture linguistique (Candelier, 2000), les étudiants démontrent une faible connaissance déclarative sur les langues (par exemple, l'anglais est signalé comme LR), qui apparaissent encore dans leurs discours comme des réalités étanches, sans rapport entre elles. Finalement, ils font part des projets linguistiques assez limités, généralement orientés vers des apprentissages à but pragmatique et professionnel et limités à un nombre réduit de langues.

26 Par rapport donc aux images du FLE, les étudiants voyaient le FLE, dans l'année 2001-2002, surtout en tant qu'objet socio-affectif, caractérisant cette langue comme étant "belle", "romantique", "chic" et "élégante". Il s'agit donc d'un rapport affectif à la langue fondé sur l'élan suscité par la langue-culture et ses locuteurs. Le français inspire chez les étudiants d'abondantes associations lexicales, notamment en rapport avec la cordialité de la vie sociale et des interactions ("bonjour", "merci", "au revoir", "salut", "bonsoir" et "de rien"). En plus, nous avons retrouvé une forte allusion à un romantisme encore associé à la France et aux Français, ce qui avait déjà été noté dans l'étude de Williams, Burden \& Kanvers (2002): "French is the language of love and stuff" (les mots évoqués étant "amour", "je t'aime", "cœur", "mon amour" et "la vie en rose").

Deuxièmement, le FLE était envisagé en tant qu'objet d'appropriation en contexte formel, les références par rapport à sa facilité ou difficulté les plus abondantes étant les suivantes : 
"proche du latin", "proche du portugais", "facile", "difficile" (voir Melo \& Araújo e Sá, 2006, pour plus de détails).

Finalement, le français est encore compris en tant qu'objet de pouvoir économique et professionnel, une langue utile et un atout dans une future carrière. Ainsi, interrogés sur la LR dont ils considèrent l'apprentissage comme le plus important en contexte portugais, la majorité des étudiants mentionne le FLE, surtout par des raisons utilitaires et pragmatiques: "le français est une langue internationale" (9 réponses), qui "facilite la communication" (3 réponses) et "l'accès au monde du travail" (3 réponses). En plus, ils considèrent que le français possède encore beaucoup de pouvoir économique et social (4 réponses).

Dans l'année 2003-2004, deux années après la première enquête, l'ordre des deux premières catégories est inversé : les apprenants envisagent maintenant le français surtout en tant qu'objet d'appropriation; le FLE en tant qu'objet affectif et en tant qu'objet de pouvoir occupe la deuxième et la troisième place, respectivement.

En ce qui concerne les images du FLE en tant qu'objet d'appropriation, les plus fréquentes tournent autour de sa facilité/difficulté et de la proximité par rapport à la LM. Ainsi, bien que la majorité des apprenants la considèrent comme une langue typologiquement proche du portugais, ils la considèrent une langue difficile à cause de la composante grammaticale (tout comme dans l'étude de Küster, 2007, auprès des lycéens allemands), considérée complexe, surtout par rapport à la conjugaison verbale.

31 Pour ce qui est du rapport affectif à la langue, nous remarquons que les expériences de contact préalables avec le FLE (en contexte formel et informel) et "le paysage sonore de la langue" (Lhote, 1995) jouent un rôle important. Par rapport au premier aspect, les étudiants font référence au plaisir provoqué par l'apprentissage du français, la sympathie ressentie par rapport à la langue et à ses locuteurs et au prestige du français. Ils personnifient la langue en tant que "belle", "romantique", "passionnante", "élégante", "avec du charme", "amusante", "séduisante" et "extravagante", lui conférant un statut symbolique et un prestige subjectif. Nous croyons que cette personnification cache un rapport métonymique langue-peuple. En plus, nous constatons que beaucoup de ces images stéréotypées correspondent à celles qui circulent dans le macro-contexte de ces apprenants : en effet, le "romantisme" et le "charme" attribués à la langue sont enracinés dans l'imaginaire sociolinguistique collectif, ce qui explique l'homogénéité des réponses par rapport à cet aspect, dans les deux années étudiées (et dans d'autres études, comme celle, déjà citée, de Williams, Burden \& Kanvers). Pour ce qui est de l'image sonore de la langue, maintenant plus faiblement représentée que dans l'année précédente, les étudiants la considèrent comme "harmonieuse" et "musicale", à "sonorité unique".

Une minorité des étudiants considère le FLE comme objet de pouvoir surtout à cause des rapports commerciaux entre le Portugal et la France, l'envisageant en tant que langue "utile", "pratique" et "nécessaire". Pourtant, pour une autre partie des apprenants, il s'agit d'une langue "fermée", incapable de répondre et de suivre le rythme de développement mondial ("accessoire", "répétitive"...). Par opposition à l'anglais, la langue considérée "moderne", le français est considéré une langue "historique", support d'une culture statique et dépassée, qui ne répond pas aux nouvelles réalités économiques. Cependant, quelques étudiants reconnaissent que connaître le français confère un statut économique et culturel, s'agissant d'une langue qu'on suppose parlée par les élites et, ainsi, d'une langue-outil d'auto 
promotion personnelle et d'acquisition d'un capital symbolique (dans le sens de Bourdieu, 1991).

Nous pouvons conclure que la majorité des images sur le FLE sont de nature positive, dans les deux années. L'école étant le lieu privilégié de contact avec cette langue, de l'aveu des étudiants, nous pouvons affirmer que les institutions scolaires et les universités, à travers leurs discours et autres matériaux de création et de légitimation d'images des langues, ont réussi à élaborer une stratégie de marketing, avec succès, autour du "romantisme", de la "beauté" et du rôle du français en tant qu'objet de pouvoir, lui conférant et cimentant une valeur utilitaire.

Le tableau 2 rend compte des pourcentages par rapport à chaque catégorie et sous-catégorie étudiée, dans les deux années académiques, résumant ainsi l'analyse déjà effectuée :

Tableau 2 - Comparaison des images du FLE dans les deux périodes analysées.

\begin{tabular}{|c|c|c|c|c|c|}
\hline Catégories & Sous-catégories & $\mid \begin{array}{c}\text { Année } \\
\text { 2001-2002 }\end{array}$ & TOTAL & $\begin{array}{c}\text { Année } \\
\text { 2003-2004 }\end{array}$ & TOTAL \\
\hline \multirow[t]{2}{*}{$\begin{array}{l}\text { FLE comme objet } \\
\text { d'appropriation }\end{array}$} & $\begin{array}{l}\text { Facilité/difficulté } \\
\text { d'apprentissage et usage }\end{array}$ & $21,3 \%$ & & $27,1 \%$ & \\
\hline & $\begin{array}{l}\text { Éloignement/proximité } \\
\text { par rapport à la LM ou autres } \\
\text { LE étudiés }\end{array}$ & $4,3 \%$ & $25,6 \%$ & $17 \%$ & $43,6 \%$ \\
\hline \multirow[t]{2}{*}{ PLE comme objet affectif } & $\begin{array}{l}\text { Rapport affectif } \\
\text { apprenant - langue - culture }\end{array}$ & $44,5 \%$ & $61,8 \%$ & $27,1 \%$ & $39,8 \%$ \\
\hline & Image sonore du FLE & $17,3 \%$ & & $12,7 \%$ & \\
\hline \multirow[t]{2}{*}{$\begin{array}{c}\text { FLE comme objet de } \\
\text { pouvoir }\end{array}$} & Pouvoir social et culturel & $0 \%$ & & $4,2 \%$ & \\
\hline & $\begin{array}{l}\text { Pouvoir économique et } \\
\text { professionnel }\end{array}$ & 12,6 & $12,6 \%$ & $11,9 \%$ & $16,1 \%$ \\
\hline
\end{tabular}

Or, si le contenu déclaratif des images par rapport au FLE paraît assez stable et homogène, le pourcentage de mots renvoyant à chaque catégorie montre un détournement quantitatif révélateur du poids curriculaire dans la valeur attribuée à la langue. En effet, nous vérifions que les images ont subi un procès de (re)configuration, ce qui démontre que les facteurs d'ordre scolaire interviennent dans le rapport du sujet aux langues.

Ainsi, une langue initialement comprise d'un point de vue affectif, acquiert un statut fortement scolaire : la perception du FLE en tant qu'objet symbolique et esthétique, lieu des affects (images qui ont conduit les apprenants au choix du français, par rapport à l'allemand), a été substituée par une image scolarisée qui envisage la langue en tant que discipline intégrant le curriculum, qui doit être évaluée et qui pèse sur le succès académique.

37 Ce changement si marqué en l'espace de deux ans ne peut que nous inspirer quelques considérations, que nous formulerons dans la partie finale de ce texte. 


\section{Synthèse et perspectives} significative, sur la construction et l'évolution des images du FLE et que les changements observés peuvent, parfois, déclencher des effets négatifs en ce qui concerne le rapport du sujet à la langue et aux motivations pour s'engager, soit dans la réalisation des tâches scolaires, soit dans des situations d'interaction avec les locuteurs francophones. Ainsi, il n'est pas étonnant que des catégories rendant compte du FLE comme construisant des rapports interpersonnels n'atteignent pas de résultats significatifs, puisque l'apprentissage semble centré sur des aspects formels de la langue, le contexte universitaire semblant ne pas aider à envisager la langue en tant que pont entre différentes communautés et ne favorisant pas la transposition des compétences acquises en milieu scolaire à des situations réelles de pratique et de partage de la langue.

41 Ainsi, nous considérons qu'il faudrait investir dans un apprentissage de décentration qui puisse mettre en valeur les autres dimensions des langues, notamment leurs valeurs formatives et humanistes, dans le sens attribué par Phipps \& Gonzalez (2004) au terme languaging. Or, de notre point de vue, ceci implique une approche des langues en contexte universitaire qui prône leur apprentissage bien au-delà des atouts professionnels et comme étant bien plus qu'un objet curriculaire (primauté de l'évaluation et des résultats des apprentissages sur le procès d'apprentissage), ce qui nécessite une reconfiguration des objectifs et des activités en classe, ainsi qu'une prise en compte des biographies sociolinguistiques des apprenants (et donc, de leur compétence plurilingue et des possibilités de transfert entre les langues qui la constituent) et des besoins de mobilité à l'heure actuelle, aussi géographique que virtuelle (voir Bono \& Melo, 2008, pour une conception de la pédagogie universitaire des langues).

Ces considérations rejoignent les principes du Cadre Européen de Référence pour les Langues, qui prône la formation de citoyens conscients de l'espace plurilingue et pluriculturel qu'ils habitent (et qui façonne leurs expériences professionnelles, personnelles, ...) et capables d'y intervenir, de façon responsable et éveillée. Ainsi, le développement d'une compétence plurilingue et pluriculturelle ne doit pas être absent du curriculum universitaire en langues, ce qui implique de penser le caractère multidimensionnel des langues (du FLE, dans le cadre de ce texte) et la pluralité des rôles qu'elles sont appelées à 
jouer, notamment en tant que médiatrices entre sujets, en situation de travail comme dans d'autres contextes.

En ce qui concerne les perspectives futures de travail, afin d'éclairer un peu plus nos résultats, nous envisageons d'analyser les conceptions et les pratiques d'enseignementapprentissage du FLE (des apprenants et des professeurs), les configurations curriculaires (surtout à l'heure des modifications introduites à la suite du Processus de Bologne) et les programmes (notamment les compétences visées et les procédures d'évaluation prévues) et finalement, le statut et les rôles accordés au français par les curricula académiques et par les politiques linguistiques des universités.

\section{BIBLIOGRAPHIE}

Andrade, A. I., Araújo e Sá. M. H. \& Moreira, G. (coord.) (2007). Imagens das línguas e do plurilinguismo : princípios e sugestões de intervenção educativa. Cadernos do LALE, Série Propostas 4. Aveiro : Universidade de Aveiro, CIDTFF.

Androulakis, G. et al. (2007). "Pour le multilinguisme : exploiter à l'école la diversité des contextes européens. Liège : Les Éditions de la Faculté de Liège”. Disponible en ligne. http://www.unigiessen.de/rom-didaktik/Multilingualism/.

Araújo e Sá, M. H. \& Melo, S. (2005). "Pour une pratique de l'intercompréhension en langues romanes : les besoins et attentes d'un groupe cible (étudiants universitaires non spécialistes en langues)". Travaux de Didactique du Français Langue Étrangère, nº ${ }^{54}$. pp. 31-55.

Araújo e Sá, M. H. ; Melo, S. \& Pinto, S. (2004). "The influence of the schooling background on learners' intercultural communicative competence - a study with students from the language and business relations course at the University of Aveiro". Communication orale à ECER 04 (The European Educational Research Association). Rethymnon, University of Crete, 20-25 septembre 2004.

Araújo e Sá, M. H. \& Pinto, S (2006). "Imagens dos outros e suas línguas em comunidades escolares : produtividade de uma temática de investigação em educação linguística". In Bizarro, R. (org.). A Escola e a diversidade cultural - multiculturalismo, interculturalismo e educação. Porto : Areal Editores.

Billiez, J. (1996). "Langues de soi, langues voisines : représentations entrecroisées". Études de linguistique appliquée, Revue de didactologie des langues-cultures, $\mathrm{n}^{\circ}$ 104. pp. 401-410.

Bono, M. \& Melo, S. (2008). "Aspects contractuels dans la gestion des interactions plurilingues en contexte universitaire". Revue Canadienne de Langues Vivantes, vol. 65, $\mathrm{n}^{\circ}$ 2. pp. 33-60.Bourdieu, P. (1991). Language and Symbolic Power. Cornwall: Polity Press.

Calvet, L.-J. (1999). Pour une écologie des langues du monde. Paris : Plon.

Candelier, M. (2000). "Les objectifs de l'éveil aux langues". In Candelier, M. (coord.). L'introduction de l'éveil aux langues dans le curriculum. Annexes au rapport d'atelier. Graz : Document polycopié. 
Candelier, M. \& Hermann-Brennecke, G. (1993). Entre le choix et l'abandon. Les langues étrangères à l'école vues d'Allemagne et de France. Paris : Didier.

Castellotti, V., Coste, D. \& Moore, D. (2001). "Le proche et le lointain dans les représentations des langues et de leur apprentissage". In Moore, D. (coord.). Les représentations des langues et de leur apprentissage. Références, modèles, données et méthodes. Paris : Didier.

Castellotti, V. \& Moore, D. (2002). "Social representations of languages and teaching. Reference study". Strasbourg: Language Policy Division, DGIV, Council of Europe. http://www.coe.int/t/dg4/ linguistic/Source/CastellottiMooreEN.pdf.

Cazals-Quenouille, M. (1995). "La séquence d'apprentissage : un lieu d'inscription de la représentation des objectifs généraux des langues vivantes". Les Langues Modernes, $n^{\circ}$ 3. pp. 11-17.

Coste, D. (1997). "Le théâtre de la représentation". In Zarate, G. (coord.). Les Représentations en didactique des langues et cultures. Notions en Question, $\mathrm{n}^{\circ}$ 2. Paris : Crédif - Didier.

Cruz, M. (2005). O cibercomunicador intercultural : imagens das linguas em chat plurilingue. Dissertação de mestrado. Aveiro : Universidade de Aveiro.

Dabène, L. (1994). Repères sociolinguistiques pour l'enseignement des langues. Paris : Hachette.

Dabène, L. (1997). "Les images des langues et leur apprentissage". In Matthey, M. (org.). Les langues et leurs images. Neuchâtel : IRDP Éditeur.

De Pietro, J.-F. \& Müller, N. (1997). "La construction de l'image de l'autre dans l'interaction. Des coulisses de l'implicite à la mise en scène". Bulletin Suisse de Linguistique Appliquée, $\mathrm{n}^{\circ} 65$. pp. 25-46.

Deprez, C. (1997). "L'apprenant et ses langues : représentations métalinguistiques dans les entretiens autobiographiques". In Zarate, G. (coord.). Les représentations en didactique des langues et cultures. Notions en Question, ${ }^{\circ}$ 2. Paris : Crédif - Didier.

Graham, S. (2003). "Learners' metacognitive beliefs. A Modern Foreign Languages case study". Research in Education, n ${ }^{\circ}$ 70. pp. 9-20.

Küster, L. (2007). "Schülermotivation und Unterrichtsalltag im Fach Französisch". Französisch Heute , no 3. pp. 210-226.

Lhote, E. (1995). Enseigner l'oral en interaction : percevoir, écouter, comprendre. Paris : Hachette.

Mariko, H. (2005). "La notion de représentation en didactique des langues". http://wwwsoc.nii.ac.jp/ sjdf/Revue/\%83t\%83\%89\%83\%93\%83X33-069-086.pdf.

Matthey, M. (1997). "Représentations sociales et langage". In Matthey, M. (org.). Les langues et leurs images. Neuchâtel : IRDP Éditeur.

Melo, S. (2006). Emergência e negociação de imagens das línguas em encontros interculturais plurilingues em chat. Thèse de doctorat. Aveiro : Universidade de Aveiro. http://www.galanet.eu/.

Melo, S. \& Araújo e Sá, M. H. (2006). "Retratos de família : imagens de bem e mal querer das línguas românicas por alunos lusófonos". In Andrade, A. I. \& Araújo e Sá, M. H. (org.). Imagens das línguas em contextos de educação e formação linguística. Cadernos do Lale, Série Reflexões, 2. Aveiro : Universidade de Aveiro.

Monnanteuil, F. (1994). "Images des pays et enseignement scolaire des langues : quelques réflexions à partir des journées d'études de L'APLV". Les Langues Modernes, n 4. pp. 5-7. 
Moore, D. (2001). "Les représentations des langues et de leur apprentissage : itinéraires théoriques et méthodologiques". In Moore, D. (coord.). Les représentations des langues et de leur apprentissage. Références, modèles, données et méthodes. Paris : Didier.

Müller, N. \& De Pietro, J. F. (2001). "Que faire de la notion de représentations ? Questions méthodologiques et didactiques à partir de travaux sur le rôle des représentations dans l'apprentissage d'une langue". In Moore, D. (coord.). Les représentations des langues et de leur apprentissage. Références, modèles, données et méthodes. Paris : Didier.

Paganini, G. (1994). "Désir d'Italie, ou la représentation d'une troisième langue". Les Langues Modernes, $\mathrm{n}^{\circ}$ 4. pp. 9-17.

Phipps, A. \& Gonzalez, M. (2004). Modern languages. Learning and teaching in an intercultural field. Londres: Sage Publications.

Pinto, S. (2005). Imagens das línguas estrangeiras de alunos universitários portugueses. Tese de Mestrado. Aveiro: Universidade de Aveiro.

Schmidt, A. \& Araújo e Sá, M. H. (2005). "Schwierig, hässlich und Würstchen: Das Image der Deutschen Sprache an Schulen in Portugal. Fremdsprache Deutsch Europäisch". Akten der zweiten internationalen Konferenz des Comenius-Netzwerks DAF-SÜDOST: Budapest. http://www.dafnetzwerk.org/tagungen/ergebnisse/konferenz2005-10/Fremdsprache_Deutsch_Europaeisch.pdf.

Tschoumy, J.-A. (1997). "Introduction : une thématique nouvelle". In Matthey, M. (org.) Les langues et leurs images. Neuchâtel : IRDP Editeur.

Vasseur, M. T. \& Grandcolas, B. (1997). "Regards croisés. Rôles, représentations et réflexion dans l'enseignement-apprentissage d'une langue étrangère : le français dans l'enseignement secondaire britannique". In Matthey, M. (org.). Les langues et leurs images. Neuchâtel : IRDP Editeur.

Verdelhan-Bourgade, M. (1999). "FLS et FLE : deux représentations différentes de la norme". Le Français Aujourd'hui, nº 124. pp. 67-77.

Williams, M., Burden, R. \& Kanvers, U. (2002). "French is the language of love and stuff: student perceptions of issues related to motivation in learning a foreign language". British Educational Research Journal, vol. 4, n² 28. pp. 503-528.

Yanaprasart, P. (2002). "La dynamique des représentations dans les contacts interculturels". In Yanaprasart, P. (ed.). Communiquer en milieu interculturel. Travaux neuchâtelois de linguistique, $\mathrm{n}^{\circ}$ 36. pp. 59-79.

Zarate, G. (1993). Représentations de l'étranger et didactique des langues. Paris : Didier.

Zarate, G. (1997). "La notion de représentation et ses déclinaisons". In Zarate, G. (coord.). Les représentations en didactique des langues et cultures. Notions en Question, $\mathrm{n}^{\circ} 2$. Paris : Crédif Didier.

\section{NOTES}

2. Cet état des lieux est aussi valable en contexte allemand (voir Küster, 2007, pour une étude concernant la "crise du français" dans les lycées allemands).

3. Soutien du projet "Imagens das línguas na comunicação intercultural: contributos para o desenvolvimento da competência plurilingue", financé par FEDER, dans le cadre de Eixo 2, Medida 2.3 do Pocti - Programa Operacional "Ciência, Tecnologia, Inovação", du QCA III. 
4. Ces catégories ont été encore reprises dans d'autres travaux de recherche (Cruz, 2005 et Melo, 2006) et sont cadrées par les études de l'équipe du projet "Images des Langues dans la Communication Interculturelle" (voir Andrade, Araújo e Sá \& Moreira, 2007).

5. Les autres images de la langue, sans grande représentativité numérique, étant : FLE comme instrument de construction de rapports interpersonnels et inter-groupaux et FLE comme instrument de construction et d'affirmation d'identités individuelles et collectives.

6. Le curriculum portugais comporte maintenant l'espagnol.

7. Il faut noter qu'au Portugal tous les programmes à la télévision sont présentés dans leur(s) langue(s) d'origine.

\section{RÉSUMÉS}

Prenant le concept d' "image" en tant qu'élément central dans l'enseignement-apprentissage des langues, ce travail cherche à cerner et à comprendre les images d'étudiants portugais à propos du FLE, dans deux moments de leur parcours académique. Nous concluons que ces images sont extrêmement scolarisées et homogènes dans les deux périodes étudiées. Cette principale conclusion nous permet de réfléchir sur le rôle du curriculum universitaire dans la construction et dans l'évolution de ces images, ainsi que sur le besoin de rendre le discours éducatif universitaire plus attentif au plurilinguisme.

Taking the concept of "image" as a central feature in the process of language teaching and learning, this article describes and attempts to understand the images of Portuguese higher education students concerning French as a foreign language, during two consecutive academic years. Our results show that these images are extremely academic and homogeneous at the two different moments of the research. This main conclusion allows us to reflect upon the role of the curriculum in the construction and evolution of those images and also upon the need to make higher education discourse more aware of plurilingualism.

\section{INDEX}

Mots-clés : images des langues, français langue étrangère (FLE), didactique des langues-cultures Keywords : images of languages, French as a foreign language, language didactics

\section{AUTEURS}

\section{SÍLVIA MELO-PFEIFER}

Sílvia Melo-Pfeifer a développé une thèse de doctorat intitulée "Emergência e negociação de imagens das línguas em Encontros Interculturais Plurilingues em chat", sous la supervision scientifique de Maria Helena de Araújo e Sá, en Didactique des Langues, à l'Université d'Aveiro. Elle travaille à présent sur un projet post doctoral au LIDILEM, Université Stendhal Grenoble 3, sous la supervision de Christian Degache. Ses intérêts scientifiques sont : la communication en ligne et ses apports à l'enseignement-apprentissage des langues, l'interaction plurilingue et 
interculturelle, la co-construction de l'intercompréhension en langues romanes et, plus récemment, l'épistémologie de la DL.

Courriel : smelo[at]dte.ua.pt

Adresse : LALE - Laboratório Aberto para a Aprendizagem de Línguas Estrangeiras, Departamento de Didáctica e Tecnologia Educativa, Universidade de Aveiro, 3810-193 Aveiro, Portugal.

Courriel : lale[at]dte.ua.pt

\section{SUSANA PINTO}

Susana Pinto développe un travail de doctorat en Didactique de Langues sous le titre "Le rôle de l'institution universitaire dans la construction d'une Europe plurilingue et interculturelle. Une étude de cas avec l'Universidade de Aveiro". Son travail est intégré au Laboratoire Ouvert pour l'Apprentissage de Langues Étrangères (LALE), du Département de Didactique et de Technologie Éducative, à l'Université d'Aveiro. Ses intérêts de recherche portent notamment sur des questions concernant l'enseignement et l'apprentissage des langues, la communication interculturelle, la compétence plurilingue et les politiques linguistiques.

Courriel : spinto[at]ua.pt.

Toile : http://www2.dte.ua.pt/lale/

Adresse: Laboratório Aberto para a Aprendizagem de Línguas Estrangeiras - Departamento de Didáctica e Tecnologia Educativa, Universidade de Aveiro. Campus Universitário de Santiago, 3810-193 Aveiro, Portugal. 